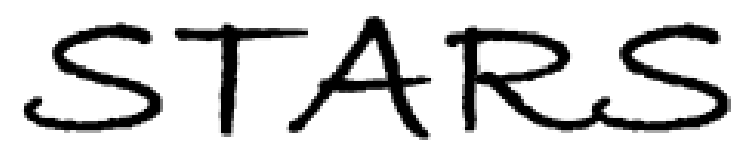

University of Central Florida

STARS

$1-1-2006$

\title{
Direct-write electron-beam lithography of an IR antenna-coupled microbolometer onto the surface of a hemispherical lens
}

\author{
C. T. Middlebrook \\ University of Central Florida \\ G. Zummo \\ University of Central Florida \\ G. D. Boreman \\ University of Central Florida
}

Find similar works at: https://stars.library.ucf.edu/facultybib2000

University of Central Florida Libraries http://library.ucf.edu

This Article is brought to you for free and open access by the Faculty Bibliography at STARS. It has been accepted for inclusion in Faculty Bibliography 2000s by an authorized administrator of STARS. For more information, please contactSTARS@ucf.edu.

\section{Recommended Citation}

Middlebrook, C. T.; Zummo, G.; and Boreman, G. D., "Direct-write electron-beam lithography of an IR antenna-coupled microbolometer onto the surface of a hemispherical lens" (2006). Faculty Bibliography 2000s. 6434.

https://stars.library.ucf.edu/facultybib2000/6434

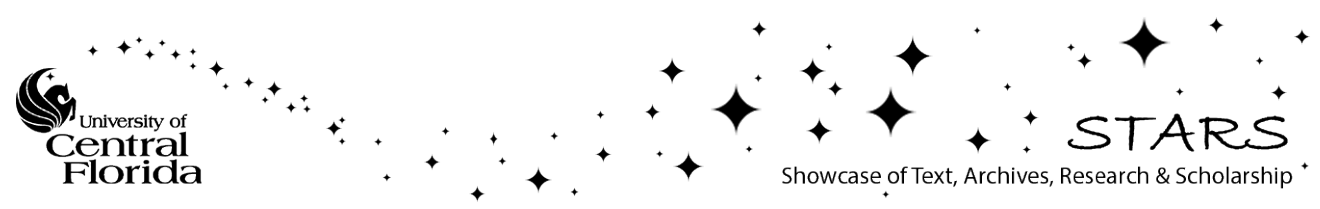


Direct-write electron-beam lithography of an IR antenna-coupled microbolometer onto the surface of a hemispherical lens

C. T. Middlebrook, G. Zummo, and G. D. Boreman

Citation: Journal of Vacuum Science \& Technology B: Microelectronics and Nanometer Structures Processing, Measurement, and Phenomena 24, 2566 (2006); doi: 10.1116/1.2360978

View online: https://doi.org/10.1116/1.2360978

View Table of Contents: https://avs.scitation.org/toc/jvn/24/6

Published by the American Institute of Physics 


\title{
Direct-write electron-beam lithography of an IR antenna-coupled microbolometer onto the surface of a hemispherical lens
}

\author{
C. T. Middlebrook, ${ }^{\text {a) }}$ G. Zummo, and G. D. Boreman \\ College of Optics and Photonics (CREOL), University of Central Florida, Orlando, Florida 32817-2700
}

(Received 12 July 2006; accepted 12 September 2006; published 31 October 2006)

\begin{abstract}
This article describes a method for performing direct-write lithography of an IR antenna-coupled microbolometer onto the surface of a hemispherical lens. Antennas on a dielectric half-space receive power more efficiently from the substrate side than from the air side. The use of a hemispherical lens facilitates reception through the substrate as well as elimination of trapped surface waves that would normally occur in the substrate. Using direct-write lithography onto the surface of the hemispherical lens eliminates the potential of an air gap between the antenna and lens. Additionally, the accuracy of alignment between the antenna and the center of the lens is controlled at the lithographic step. As a result, there is increased responsivity is observed in the antenna-coupled microbolometer when illuminated from the substrate-side compared to air-side illumination. (C) 2006 American Vacuum Society. [DOI: 10.1116/1.2360978]
\end{abstract}

\section{INTRODUCTION}

Antenna-coupled microbolometers allow for detection of infrared (IR) radiation. ${ }^{1}$ Incident radiation induces IRfrequency current waves into the antenna arms which are then dissipated in the microbolometer. This causes a change in resistance proportional to the incident radiation. Fabrication of antenna-coupled microbolometer devices is accomplished using direct-write electron-beam lithography on a silicon substrate. Antennas on a dielectric half-space will radiate or receive power more efficiently from the substrate than from the air. ${ }^{2}$ In order to take advantage of this gain factor, optimum antenna illumination occurs through the substrate. A difficulty that arises when performing substrate-side illumination is the excitation of trapped surface waves. The physical origin of this phenomenon can be visualized if we think of the antenna as transmitting. Then, any rays leaving the antenna at greater than critical angle will be completely reflected and trapped as surface waves. To overcome this problem it is desirable to use a hemispherical immersion lens where the antenna is located at the center of curvature. Matching the incoming radiation's radius of curvature to the spherical side of the hemispherical lens results in the rays entering the lens normal to the surface. Therefore, the antenna can still radiate through the substrate while eliminating the surface modes. Precise alignment of the antenna to the center of the hemispherical lens is crucial and can present difficulties when performing this alignment mechanically. Additionally, at IR frequencies it is difficult to avoid an air gap between the hemispherical lens and the substrate, which causes refraction and multiple surface reflection losses. Use of an index-matching fluid might be possible to correct this situation, but there are none readily available that accurately match silicon's permittivity with good IR transmission. Performing direct-write lithography onto the surface of the hemispherical lens allows accurate alignment between the

${ }^{a)}$ Electronic mail: cmiddle@creol.ucf.edu device and the center of the lens to be achieved. Fabrication in this way also eliminates the possibility of creating an air gap, allowing the optimum illumination condition to be obtained.

\section{FABRICATION}

The hemispherical lens used is a high resistivity $12.5 \mathrm{k} \Omega \mathrm{cm}$ silicon lens with a $10 \pm 0.1 \mathrm{~mm}$ diameter, a thickness of $5 \pm 0.1 \mathrm{~mm}$, and a surface quality of $80 / 50$ (scratch/dig). In order to perform electron-beam lithography on the lens, the flat side must first be coated with e-beam sensitive resist. To facilitate spinning the hemispherical lens a special holder was constructed that held the hemispherical lens in place while it was spin coated, which is shown in Fig. 1(a). A rubber gasket was used to form a seal to the outer portion of the lens's convex side which allowed a vacuum to be created that kept the lens in place under rotation. The hemispherical lens was then coated with bilayer resist. The bottom layer was a $350 \mathrm{~nm}$ of polymethyl methacrylate-methyl acrylic acid $9 \%$ baked for $10 \mathrm{~min}$ on a hot plate at $180^{\circ} \mathrm{C}$. The top layer was $150 \mathrm{~nm}$ of $950 \mathrm{~K}$ polymethyl methacrylate baked for $10 \mathrm{~min}$ on a hot plate at $180^{\circ} \mathrm{C}$. In order to bake the solvent from the resist, a second special holder was constructed, which is shown in Fig. 2(b). This holder kept the hemispherical lens approximately horizontal during the bake process. By only making physical contact with the outer portion of the lens's convex side, scratching of the spherical portion of the lens was prevented.

In order to facilitate loading of the hemispherical lens into the electron-beam writer, a special chuck, shown in Fig. 3, was manufactured, allowing the hemispherical lens to be held securely in place while keeping the flat surface at the correct working distance. A retaining ring was employed to keep the lens secure and level, and to provide electrical grounding for charging effects.

The most problematic portion of the hemispherical lens fabrication process was to accurately center the antenna on the ball lens while it was being written. The first attempt at 


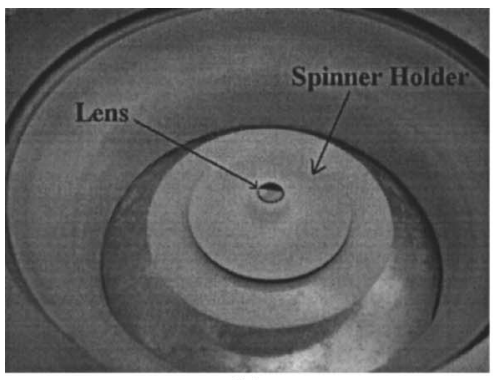

(a)

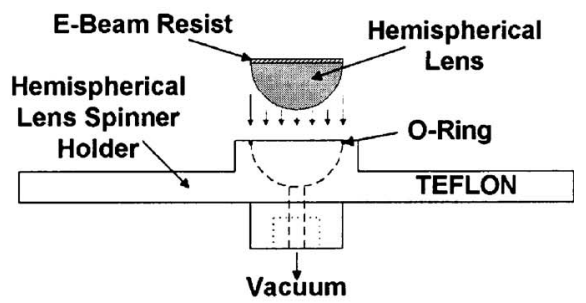

(b)
FIG. 1. (a) Image of the spinner holder for the hemispherical lens. (b) Drawing of spinner holder for the hemispherical lens. locating the center of the ball lens used the measurement of four machined marks in the retaining ring of the holder (seen in Fig. 3). Using these measurements, the center of the lens was determined by the intersection of two lines. The e-beam stage was then driven to this calculated center and the antenna was written. The measurement results of the first device fabricated showed no increase in antenna gain between the air-side and substrate-side measurements (see Sec. III below). It was suspected that no gain increase was seen in this case because the antenna was not directly in the center of the hemispherical lens. The accuracy of antenna placement using this method is estimated to be within $\pm 30 \mu \mathrm{m}$ of the of the lens's center.

In an attempt to improve antenna placement accuracy a different method that did not rely on the scribed alignment marks in the retaining ring was used to determine the center of the ball lens. The center of the ball lens was determined by measuring and determining the coordinates of the top, bottom, and sides of the retaining ring. The center of the lens was determined using the geometrical mean between these measurements. Unfortunately, this second method of using the inner circumference of the retaining ring still did not prove accurate enough to show any increase in gain when the antenna was measured. The accuracy of antenna placement using this method is estimated to be within $\pm 20 \mu \mathrm{m}$ of the lens's center. Since the above calculations involved in determining the center of the ball lens relied upon the accuracy of the machined inner circle of the retaining ring, it was thought that removal of the ring as a reference in the measurements would eliminate centering inaccuracies. Therefore, the next attempt at finding the center of the lens was attempted without the retaining ring in place. This approach using the measurements of the actual top, bottom, and sides of the hemispherical lens was used in place of the retaining ring measurements to determine the center of the lens and re- sulted in better accuracy which is discussed more in Sec. III. The accuracy of antenna placement using this method is estimated to be within $\pm 10 \mu \mathrm{m}$ of the lens's center. Further studies are being conducted in order to achieve a better alignment procedure to determine the exact center of the hemispherical lens, as well as measurement of the complete antenna angular patterns ( $E$ plane and $H$ plane) in order to indicate exactly how far the device placement deviates from the center.

Once the center position of the ball lens was determined, the resist was patterned using a modified JEOL 5900 scanning electron microscope (SEM) with $\mathrm{a} \mathrm{LaB}_{6}$ cathode. The cathode was operated at an accelerating voltage of $30 \mathrm{kV}$. Raith ELPHY QUANTUM software was used to manipulate the electron beam. The antenna and the lead lines were exposed as areas in a $100 \times 100 \mu \mathrm{m}^{2}$ write field with an area dose of $100 \mu \mathrm{A} \mathrm{s} / \mathrm{cm}^{2}$, a dwell time of $3 \mu \mathrm{s}$, an area step size of $0.003052 \mu \mathrm{m}$, and an exposure current of $56 \mathrm{pA}$. The bond pads were then exposed in a $700 \times 700 \mu \mathrm{m}^{2}$ write field with an area dose of $100 \mu \mathrm{A} \mathrm{s} / \mathrm{cm}^{2}$, a dwell time of $3 \mu \mathrm{s}$, an area step size of $0.032043 \mu \mathrm{m}$, and an exposure current of $650 \mathrm{pA}$. Following the exposure, the resist was then developed for $60 \mathrm{~s}$ in a 1:3 mixture of methylisobutylketone:isopropanol.

In order to perform metal deposition for the antenna, a special mounting apparatus to invert and suspend the hemispherical lens in an evaporation system was designed, as shown in Fig. 4. The holder was constructed of brass in order to provide a good conduction path to the actively watercooled block already in place in the evaporator. A $150 \mathrm{~nm}$ layer of $\mathrm{Au}$ was evaporated onto the patterned resist using a BOC Edwards Auto 306 electron-beam evaporation system. The rate of deposition was $0.8 \mathrm{~nm} / \mathrm{s}$ with a beam current of $80 \mathrm{~mA}$ and an accelerating voltage of $4.5 \mathrm{kV}$. The hemispherical lens was then placed into methelyne chloride, and

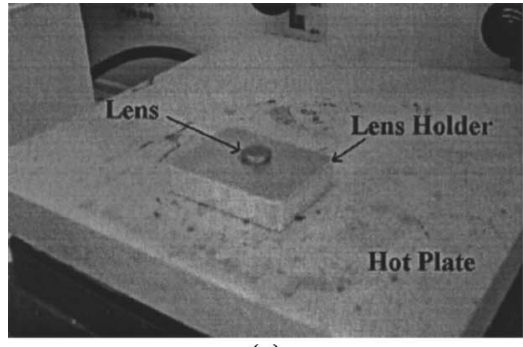

(a)

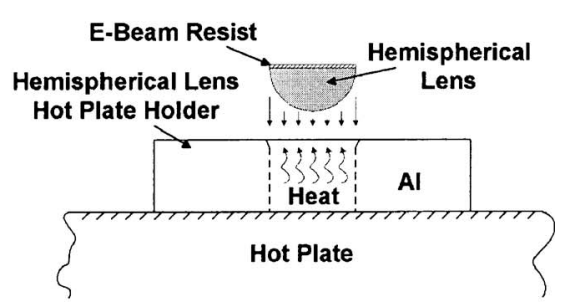

(b)
FIG. 2. (a) Image of the hot-plate holder for the hemispherical lens. (b) Drawing of the hot-plate holder for the hemispherical lens. 


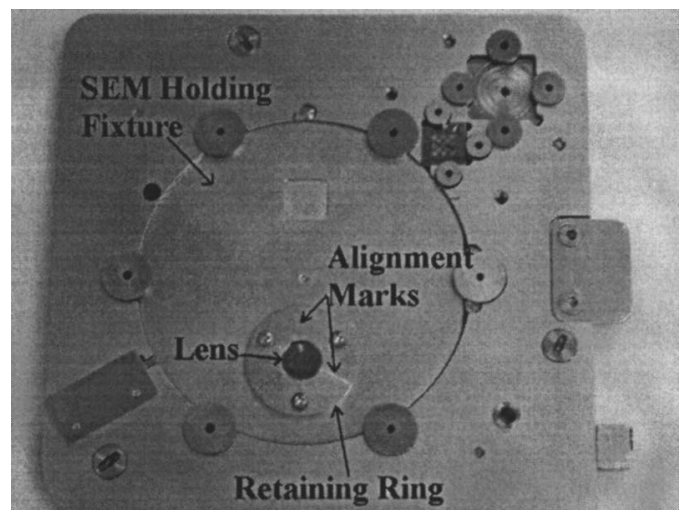

FIG. 3. Image of the hemispherical lens and SEM fixture.

ultrasonic agitation was used to remove the remaining resist and excess metal. The hemispherical lens was cleaned, and the process above was repeated for the patterning of the bolometer. A $100 \mathrm{~nm}$ layer of vanadium was used as the bolometer material and was sputtered using a MRC 8667 sputtering system. dc magnetron sputtering of vanadium was completed using an $\mathrm{Ar}^{+}$plasma with a chamber pressure of 4.5 mtorr, a dc voltage of $400 \mathrm{~V}$, and a power of $2 \mathrm{~kW}$ for a deposition rate of $2 \mathrm{~nm} / \mathrm{s}$. A lift-off procedure was again used to remove the remaining resist and excess metal. Figure 5 shows a SEM image of the completed antenna and bolometer at the center of the lens.

\section{TESTING}

Testing of the devices consisted of measuring both the air-side and the substrate-side responsivity of the antenna. In order to eliminate any specific device differences, a special chip carrier was built for this application that allowed for the use of the same device for both measurements. The chip carrier allowed illumination from both sides (air and substrate), as shown in Fig. 6, without having to remove the ball lens. Once the air-side illumination data were obtained, the chip carrier was simply reversed and the substrate-side data were taken. At IR frequencies the coupling efficiency $\left(\Gamma_{\varepsilon}\right)$ of an antenna will be $\sim 40$ times more efficient for radiation incident from the substrate than from the air side, ${ }^{3-5}$

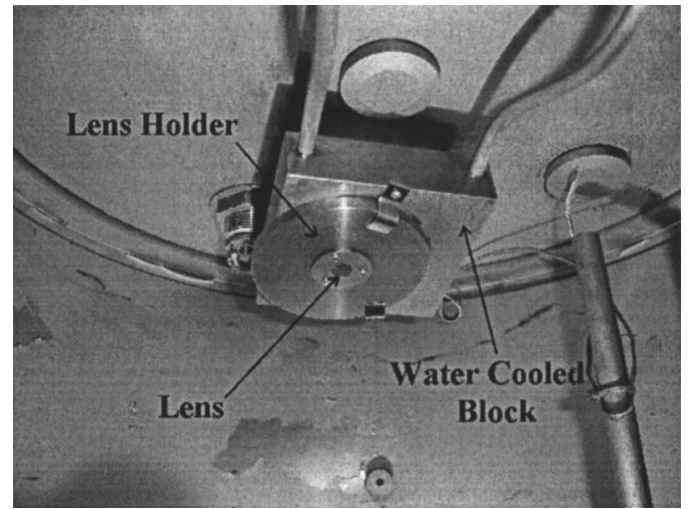

FIG. 4. Image of the hemispherical lens and evaporator holder.

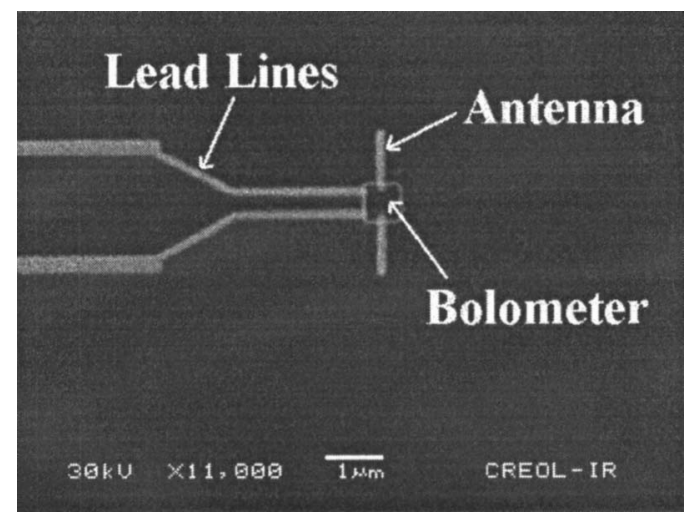

FIG. 5. SEM Image of the finished antenna-coupled microbolometer at the center of the lens.

$$
\Gamma_{\varepsilon}=\frac{\Phi_{\mathrm{Si}}}{\Phi_{\mathrm{air}}} \approx\left(\frac{\varepsilon_{\mathrm{Si}}}{\varepsilon_{\mathrm{air}}}\right)^{3 / 2} \approx 40 .
$$

Therefore, a significant gain is expected (even taking into account reflection loss at the spherical lens surface) between the substrate-side and air-side illumination conditions.

The measurement results of the first two devices fabricated showed no increase in gain between the air-side and substrate-side measurements. It was suspected that no gain increase was observed because the antenna was not in the true center of the hemispherical lens. Measurement of the angular patterns verified this suspicion. The measured angular pattern of the substrate side revealed that the antenna had a larger response off-normal in one of the rotational directions. This would correspond to an antenna being located off-center and out of the focused spot. ${ }^{6}$ When the device was rotated for angular pattern measurement the center of rotation was not based on the geometric center of the antenna, but rather on the center of the lens.

In the case of the where the lens center was determined using the measurements from the actual sides of the lens, the accuracy had improved from previous attempts, showing an increase in gain from the substrate-side versus air-side illumination. Even though the measured angular pattern still indicated that the device was not positioned at the exact center of the lens, the improved accuracy placement of the device allowed for a gain factor of 11.7 in terms of responsivity obtained. Taking into account the Fresnel reflection loss at the air-silicon boundary with $n_{\mathrm{Si}}=3.4$ and air assumed to be $n_{\text {air }}=1.0$, there is $29.7 \%$ of the incident radiation that is lost upon reflection. When reflection is taken into account, the gain factor in responsivity between the substrate-side illumination and air-side illumination is 15.2.

\section{SUMMARY}

By means of direct-write lithography onto the planar surface of a hemispherical lens, an increase in the responsivity of an IR antenna-coupled microbolometer illuminated through the substrate has been achieved. The fabrication process is a standard approach, but utilizes several novel holders 


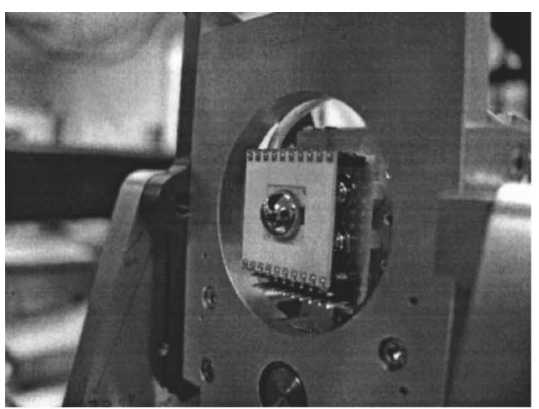

(a)

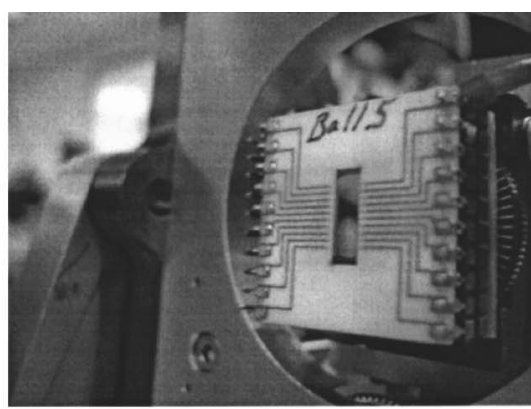

(b)
FIG. 6. Image of the hemispherical lens mounted on a chip carrier for (a) substrate-side illumination and (b) airside illumination. and fixtures tailored to this specific application. Various techniques of determining the center of the hemispherical lens using the SEM were explored. Comparison of the substrateside responsivity and air-side responsivity revealed a gain factor of 11.7 between the two measurements or 15.2, taking into account Fresnel reflection losses. This gain is due to the antenna being better matched to the substrate side than to the air side, allowing for greater responsivity of the antenna through the substrate. Using reciprocity, this translated into the antenna being able to receive more power through substrate-side illumination than air-side illumination.

Further research is currently being conducted to determine a more reliable way of finding the exact center of the hemispherical lens. More precise alignment between the antenna and the hemispherical lens will further increase the gain factor reported in this article.

${ }^{1}$ I. Codreanu, F. J. González, and G. D. Boreman, Infrared Phys. Technol. 44, 155 (2003).

${ }^{2}$ D. B. Rutledge, D. P. Neikirk, and D. P. Kasilingam, Int. J. Infrared Millim. Waves 10, 1 (1983).

${ }^{3}$ C. R. Brewitt-Taylor, D. J. Gunton, and H. D. Rees, Electron. Lett. 7, 729 (1981).

${ }^{4}$ M. Kominami, D. Pozar, and D. Schaubert, IEEE Trans. Antennas Propag. AP-33, 600 (1985).

${ }^{5}$ J. Alda, C. Fumeaux, M. Gritz, D. Spencer, and G. Boreman, Infrared Phys. Technol. 41, 1 (2000).

${ }^{6}$ D. Filipovic, G. Gauthier, S. Raman, and G. Rebeiz, IEEE Trans. Antennas Propag. 45, 760 (1997). 